\title{
STUDYING DESIGN THINKING AS A FORTHCOMING SOURCE TO INNOVATION SPEED
}

\author{
Mitcheltree, Christina Marie; Holtskog, Halvor; Ringen, Geir \\ NTNU
}

\begin{abstract}
With complex technology-intense industries follows an ever-increasing need for rapid innovation processes. Yet, innovation speed and the time from idea to product realization can vary and be unpredictable.

Design Thinking (DT) is suggested as a key driver to impact the speed of product innovation within product development projects. To understand and aid the road from early ideas and concepts to valueadded products, this paper will provide a literature study on how Design Thinking can facilitate improved product innovation performance through innovation speed.

The paper seeks to develop an overview of new insight on DT applicability for improved product innovation capability. This is done by identifying components that comprise DT's innovative ability and appropriateness to product development contexts beyond the early creative phases of product development.

As DT emphasize on visualization and re-framing problems, it contributes to enhanced clarity, meaning and confidence in ideas and decisions. DT in this way may impact strategy formulation and speed up complex innovation processes by pre-experiencing future situations.
\end{abstract}

Keywords: Design methods, New product development, Innovation, Communication, Visualisation

\section{Contact:}

Mitcheltree, Christina Marie

NTNU

Mechanical and industrial engineering

Norway

christina.mitcheltree@ntnu.no

Cite this article: Mitcheltree, C.M., Holtskog, H., Ringen, G. (2019) 'Studying Design Thinking as a Forthcoming Source to Innovation Speed', in Proceedings of the 22nd International Conference on Engineering Design (ICED19), Delft, The Netherlands, 5-8 August 2019. DOI:10.1017/dsi.2019.242 


\section{INTRODUCTION}

Design Thinking has increasingly been identified as a creative way to innovation capability. It has mainly been relevant to problem framing and idea generation in the early phases of innovation. However, it is unclear from the literature whether DT is applicable to innovation speed in later stages of product innovation processes, hence overcoming innovation barriers.

This study emphasizes on Design Thinking as a forthcoming source to innovation speed. Design Thinking (DT) as a methodology is known as a mindset and a collection of tools to achieve product or service innovation (Liedtka, 2014; Tschimmel, 2012; Carlgren, 2016; Brown, 2008; Meinel et al., 2011). The goal is to analyze research to acquire a greater level of understanding of DT applicability to speed within product development. Speed in this sense is related to looking at the DT conditions in which time may be reduced. Hence, DT tools may enable, or hinder action taken within a project depending on context.

Speed from a process innovation perspective in today's society is interesting as on the one hand, smart manufacturing processes (e.g. industry 4.0) are expected to operate rapidly to create value. However, poorly made decisions and actions may delay the process. Establishing rapid innovation processes that contribute to enhanced performance is thus important.

The purpose of this paper is to address DT's usefulness as a valid tool to address innovation speed for planned processes in later innovation phases. Hence, the question: How can Design Thinking impact the speed of product innovation performance? The paper concludes that as DT provides a creative way to product and service development, it reveals valuable insights that is applicable to strategy formulation and hence innovation performance in organizations. However, the extent to which capability is generated may rely on the combination of various DT techniques, contextual factors, and whether DT is combined with other methods. Hence, assessing organizational barriers to speed may in combination with DT provide the best output. The paper thus provides a basis for further empirical research and discussion.

\section{METHODOLOGY}

This paper has followed Cooper (1986) research stages in conducting a literature review, and involves problem formulation, data collection, data evaluation, analysis and interpretation, and public presentation. Furthermore, the review is classified according to the following characteristics (Cooper, 1988): focus (research outcomes, research methods, theories, practices/applications), goal (integrate/generalize findings, resolve debates, bridge languages across fields, critically analyze previous research, identify issues, explicate a line of argument within a field), perspectives, coverage, organization and audience.

The focus of the review is outcome based, which according to Cooper (1988) is based on findings, summarizing the literature substance and drawing conclusions. For the problem formulation stage, this involves questions guiding the review and inclusion/exclusion criteria. The paper has followed Wallace and Wray (2011, p.153) framework for critical synopses and analyses of multiple literature texts, creating a comparative critical review from completed analyses. From the synopses, associated critical analysis questions were asked.

In relation to the coverage, inclusion/exclusion criteria, and for the data collection stage, Cooper (1988) selection approach of "purposive sample" is taken. Hence, examining only central and pivotal articles within the DT field related to DT's applicability to innovation speed and efficiency within an organizational setting. DT literature was found to concentrate on the early phases of innovation, largely in relation to idea and concept generation. It was therefore a limited number of articles in the field related to DT where a connection could be made to innovation speed in later phases of product innovation. The literature chosen for analysis has been selected as the top five most relevant within DT literature to address the research question. However, to provide a solid definition of DT as a method and its challenges, other literature has been included in some parts of the review. The literature resulted from keyword searches in the academic databases Wiley and Google Scholar, and the articles chosen for in-depth analysis was chosen based on a publishing range of six years. Wallace and Wray (2011) framework is also used for the data evaluation, data analysis/interpretation and presentation stages (presenting data) of the review. However, as the authors stress that their structure is rather inflexible, there does not need to be one fixed sequence to the analysis. This paper has therefore taken 
a more flexible approach; drawing on some material from the critical analyses and synopses, but not all of it and not in any fixed sequence. The reason for applying the framework of Wallace and Wray (2011, p.153) is that it provided a good structure to the paper. This is because it created common themes of DT characteristics from comparing central arguments from key articles in the field of DT. Moreover, as the discussion does not go in depth on each DT technique, a categorization of DT characteristics could be made from the literature. Hence, a focus is placed on the three aspects: visual, practical and contextual factors of DT. The choice to divide the discussion into three aspects is based on an identification of similar arguments from within the literature chosen.

\section{RESULTS}

\subsection{Focus areas}

To answer the research question the literature review is divided into six focus areas. The focus areas were selected as the most relevant to answer the research question and were derived from the literature chosen for the analysis:

- $\quad$ The Design Thinking concept

- DT experimentation and visualization

- Tangibility through prototyping

- Collaborative characteristics of DT

- $\quad$ DT and uncertainty

- Mixing methods and DT in later phases

\subsection{The design thinking concept}

Design Thinking (DT) is mentioned as a powerful methodology for innovation that integrates human, business and technological factors in problem forming, solving and design "Design Thinking" (Meinel et al., 2011). It emphasizes on user needs, multidisciplinary collaboration and iterative improvement in producing innovative products, systems and services through rapid prototyping.

One of the first DT process models was of Simon Herbert (1969) and consists of seven phases (define, research, ideate, prototype, choose, implement, and learn) (Simon, 1996). Today, there are a variety of DT models ranging from three to seven phases. However, they all share many of the same principles. For example, DT is described as a structured process of exploration for ill-defined problems (Mahmound-Jouini et al., 2016) and a human centered innovation process that emphasize on observation, collaboration, fast learning, visualization of ideas, rapid concept prototyping, and concurrent business analysis (Lockwood, 2010). Brown (2008) argues that it is a discipline and thought process that aim to use design principles, methods and tools within management and business strategy and that it can be incorporated in all process levels.

As DT started out to improve the process of designing tangible products, it has the potential to improve the management of intangible challenges involved with engaging people with the adoption of new innovative ideas and experiences (Brown and Martin, 2015). Furthermore, as wicked or "illproblems" are addressed of being too difficult to solve with analytical methods (Buchanan, 1992), the DT process as a result, is mentioned as a "co-evolution of solution and problem space" (Cross, 2011). Hence, emphasizing on problem setting rather than problem solving. Problem framing, and visualization thus set DT apart from other management concepts (Carlgren, 2016; Liedtka, 2014), in this way impacting company strategies. However, little research exists on strategic contributions of DT on innovative project management on the firm level (Liedtka, 2014).

An important part in the history and thus future of DT is the HPI (Hasso-Plattner-Institute) and Stanford University Design Thinking Research Program. It engages multidisciplinary research teams to investigate the phenomena of the innovation method of DT in technical, business and human aspects and the impact on performance. The DT model of the Hasso-Plattner-Institute of Design at Stanford represents the common DT process and consist of a series of five major stages: (re) Defining the problem (Emphasize), Needfinding and benchmarking (Define), Brainstorm (Ideate) or Bodystorm (e.g. physically experiencing a situation to create empathy and new ideas when sketching is not adequate), Prototype (Build) and Test (Learn) (Meinel et al., 2011; Weinreich, 2011). 


\subsection{DT experimentation and visualization}

The DT approach emphasize on using visual images to impact individual's perception (Tschimmel, 2012). This derives from the understanding that DT tools, due to their visual ability, constitute clarity. Hence, making it easier for individuals within interdisciplinary teams to understand each other better. In this way, DT aid creative and collaborative processes (Tschimmel, 2012).

Seidel and Fixson (2013) mentions three formal methods within DT: Needfinding (embracing a problem definition or opportunity through observation, empathy and user understanding), Brainstorming (a formal framework for ideation), and Prototyping (building models to facilitate the development and selection of concepts). Brainstorming is mentioned as one of the main DT methods and accentuates on experimental approaches. It relates to a group process applying techniques that promote the search for new solutions. Hence, creating a structured environment to build on team members' ideas (Seidel and Fixson, 2013). Tschimmel (2012) argues that the method of brainstorming from a DT perspective can be classified in to the variants "Brainwriting" and "Brainsketching". Hence, connecting brainstorming to collective processes where visual artifacts such as Post-it's are used. Predominance of verbal communication and presentation of thoughts out loud may from more classical ways of brainstorming be replaced by rapid visualization of ideas through drawings. This help with thinking more intuitive and flexible, assisting organization and categorization of ideas (Tschimmel, 2012). Seidel and Fixson (2013) studied high and low performing novice multidisciplinary student teams within the concept generation and concept selection phases of a product innovation project. Here, they found that brainstorming as a tool for needfinding, proved to be useful to promote the search for new solutions that would be hard through individual ideation. Moreover, brainstorming can be used as a tool to solve concept related challenges. However, in their study they found limits to brainstorming effectiveness, as less successful teams had more brainstorming sessions on average and were spending brainstorming sessions in unproductive ways. As increased brainstorming sessions impact team effectiveness negatively, the authors did not know whether an increased number of brainstorming sessions was due to having difficulties with practicing it or not. Moreover, type of assignment, phase of development and team composition was argued to impact brainstorming efficiency. Nevertheless, ensuring sufficient guidance of using design methods within organizations is thus suggested (Seidel and Fixson, 2013).

As brainstorming could lead to better solutions towards concept realization, it may provide challenges in terms of conflict and debate (Seidel and Fixson, 2013). Conflict in this sense is connected to communication in innovation projects consisting of members from a range of disciplines with various perspectives. Teams thus need to find forms of communication for efficient task work (save time). However, such ways of communication with the use of DT methods may not be explicitly covered (Seidel and Fixson, 2013). The authors distinguish between process, task and relationship conflict, where task conflict can affect team performance positively. However, for relationship conflict, the opposite was true. The various terms of conflicts are not explained, but the authors refer to Jehn et al.'s (2008) definition of conflict is not related to DT. Here, task conflict is related to conflict over the content or goal of the task. Relationship conflicts are described as disagreements and incompatibilities among group members regarding personal issues that are not task-related. Process conflict however, is described as disagreements about logistical and delegation issues such as how task accomplishment should proceed in the work unit, who's responsible for what, and how things should be delegated. Seidel and Fixson (2013) focuses attention on the process aspect, relating process conflict to the process of brainstorming. Here, conflict may develop due to teams shifting concepts by replacing various elements in response to newly arrived information. Conflict (understood as disagreement) regarding establishing a concept and the process to follow may also result in debates about later changes (Seidel and Fixson, 2013). Liedtka (2014) studied decision maker cognitive bias as a challenge for innovation. The first bias category consists of the following factors:

- Overestimation of a possible future experience of an event and it being similar to a current experience (projection bias)

- Personal preferences (egocentric empathy gap)

- Decision-makers' present state impact their assessment of an idea, resulting in under- or overvaluing ideas (hot/cold gap)

- Overreacting to specific stimuli and ignoring others (focusing illusion) 
To address the projection bias, the researcher suggests developing perspective taking skills (understanding and adopting viewpoints of others) as well as the ability to imagine others' experiences. The author mentions ethnography as a need finding tool to understand others' past, perspectives, preferences and emotional state. This resembles Seidel and Fixson (2013) need finding method, however, to understand users' current situation and needs to create solutions, Liedtka (2014) suggest Job-to-be done analysis as a tool. This tool asks customers what they aim to accomplish in a relevant situation, asking them to rethink an experience, describing their thoughts, reactions, and satisfaction in every step. Hence, facilitating identification of needs not easily articulated. Being part of the user experience will make decision makers less likely to solely look at their own past experiences as the source of new ideas (projection bias), nor focus on their present state when assessing ideas (hot/cold gap). This thus fosters more valuable ideas and a wider attention focus (Liedtka, 2014).

Visualization methods like storytelling or creating metaphors or analogies through imagery (visual or narrative) on Post-it notes, or whiteboards increase imaginative abilities (Liedtka, 2014). The author argues that storytelling rather than presenting data encourage decision makers to attend and make sense of data that would otherwise be missed. Combined with ethnography, it thus improves the degree of novelty and value of generated ideas due to a focus on details in the lives of those they seek to create value. Moreover, the author argues that metaphors can guide future decisions from making sense of past and present experiences. This thus reduces reliance on the past (projection bias), widening vision (avoid focus illusion), thus recognizing various preferences to create novel and valuable ideas (empathy gap).

Carlgren (2016) looks at the DIA (Discovery-Incubation-Acceleration) framework of O'Connor and Ayers (2005) for describing innovation competencies for including the whole innovation process, not just the early stages. The framework is used as a guide for the DT method. The Discovery phase (exploration) involves user research, ethnography/video-ethnography, interviews, journey-mapping, analogies (studying similar problems but in different contexts) and digital journaling (documenting and submitting daily experiences through digital videos and text) (Carlgren, 2016). By using visual tools in interviews like covering large walls with photos, drawings, Post-its and transcripts to better frame the problem, the author stresses that deeper insights can be retrieved through reframing problems and articulation of initial ideas, thus creating meaning.

Other verbal and visual tools used in DT are the Mind map and Storyboard (Tschimmel, 2012). The Mind map consists of keywords that can be associated with other words and images to gather ideas and information. It consists of labeled twigs and branches, which represents relationships. A Storyboard on the other hand, consists of a set of images or Post it's (drawings, illustrations or photographs), shown to visualize a process or service. It is helpful in relation to elaborate a concept or testing users' interactions with a new product, service or business model.

\subsection{Tangibility through prototyping}

Tschimmel (2012) claims that DT may be used for visualizing and testing new solutions. As acceptance of failure and mistakes are significant factors of DT, rapid prototyping with cheap materials can permit early failure. Having to cope with incomplete information and unpredictability is thus preparing designers to tackle uncertainty. Hence, DT facilitates proactiveness, drives out fear and creates a tolerance for trying and failing (Tschimmel, 2012). In contrast to using prototyping to validate an idea, Seidel and Fixson (2013) argues for prototyping being especially in the earliest phases of product development, a method to stimulate the imagination. This was valuable in relation to both the concept generation and selection phase. Liedtka (2014) however, suggest using prototyping tools to improve customers' ability to identify and assess their own needs. Prototyping techniques thus helps making abstract ideas tangible as well as create "vivid manifestations" of the future though accurate feedback. Prototyping can in this way reduce a "say/do gap" by enabling customers/users to accurately describe their needs, making it easier to develop value-creating ideas for them. A say/do gap is described as a difficulty in articulating future needs as well as giving feedback on new ideas. This therefore limits decision makers in creating valuable ideas for their users (Liedtka, 2014). This is similar to Mahmound-Jouini et al. (2016) claim of using rapid prototyping to create demonstrators that enables effective dialogue and understanding to reveal unstated needs and expectations of stakeholders. 
To get the most out of workshops, Tschimmel (2012) states that team members can prepare analogous examples and personas (fictive persons) from user research. This thus helps to develop ideas on small cards for how a possible technology could be useful. Personas is also mentioned to be used as a tool for understanding end user's problems and perspectives by exemplifying a personal idea of users. Additionally, the author suggests an Empathy Map to visualize the information acquired from Personas and/or through observation and interviews. Carlgren (2016) emphasize on acquiring valuable feedback from users beyond asking questions. Team members can thus be encouraged to give feedback through spontaneous prototyping and brainstorming in relation to different idea cards. Hence, adding on Post it's with text and images as well as role-playing specific situations. This thus contributes to development and clarity of solutions through constructive dialogue and activity.

\subsection{Collaborative characteristics of DT}

DT tools are essential to enable designers to inquire about future situations or solutions to a problem (Tschimmel, 2012). In this way, the DT managers' techniques differ from the traditional managers, in that traditional managers mainly use verbal communication, diagrams and tables. In contrast, DT tools quicken and facilitate thinking processes through visualization of collected information about a project.

Mahmound-Jouini et al. (2016) mention DT as having three perspectives: cognitive (creative/explorative activity of design), organizational perspective (stakeholders involved in the design process) and strategic perspective (the strategic process of organizations and managerial capability). They address the importance of identifying and involving relevant stakeholders in the upstream phase of complex and uncertain projects to avoid drifts of projects. Developing empathy as well as using DT tools like visualization, ethnographic approaches, journey mapping and personae characterization, help players better imagine and apprehend experiences of stakeholders. Hence, mitigating the "say/do gap" mentioned by Liedtka (2014).

\subsection{DT and uncertainty}

Mahmound-Jouini et al. (2016) argues that as organizational decisions involve uncertainty and ambiguity, DT can be a benefit for intermediate organizational problems as analytical thinking is not enough for messy and ill structured situations.

The authors argue that as DT tools support deep data collection (understood as user related insights) and idea generation, it is an effective way to frontload problem and risk detection. To reduce uncertainty, learning, knowledge acquisition and identification through hypothesis articulation and experimentation is thus crucial (Mahmound-Jouini et al., 2016). Liedtka (2014) category three bias relates to flaws in decision-makers' hypothesis testing abilities. It may relate to the context of product innovation uncertainty as it involves over optimism (the planning fallacy), inability to see disconfirming data (hypothesis confirmation bias), attachment to early solutions (endowment effect), or preference for the easily imagined (availability bias). By prototyping, in this way experience ideas based on user's feedback, may thus reduce availability bias by helping innovators as well as customers to imagine novel ideas more easily (Liedtka, 2014). Moreover, experiencing failure in advance through prototyping, where a focus is put on potential future failure factors, may impact people to put more effort in the task by being mentally prepared. By experiencing products or services in advance and evaluating multiple hypothesis (Optionality), it is possible to provide several predictions and options for the future. Moreover, it is possible to describe expectancies, individual assumptions with new ideas, and identify what the data that supported or nullified the assumptions might look like (Liedtka, 2014). Nonetheless, it makes way for reflection of experiments (success/failure) improving future performance (Liedtka, 2014).

\subsection{Mixing methods and DT in later phases}

Seidel and Fixson (2013) states that successful teams combine methods, such that it is not the quantity of brainstorming sessions but their linkage to other methods (formal and informal practices) that matter. Group or team reflexivity refers to the degree to which individuals collectively reflect upon their actions and processes (Seidel and Fixson, 2013). Team reflexive practices such as reflecting and debating over ideas, processes and changes in combination with brainstorming and/or prototyping is mentioned to increase team performance. Reflective practices that constitute reflection and debate may therefore enable or constrain conflict. Conflict in this way may thus be understood as a result of not 
being able to communicate through reflective practices in a manner that substantiates DTs solutionfocused thinking. However, continuing to question ideas or a process from the concept generation to the selection phase showed itself to be inefficient (Seidel and Fixson, 2013). The authors thus suggest moving from reflexive to less reflexive behavior in the concept selection phase.

Liedtka (2014) mentions mixing DT with other literature; (team learning) and positive affects as to why DT works. Additionally, Carlgren (2016) stresses mixing methods, however she questions DT's perception that innovations involving complexity are difficult to fit in a measurement culture. The term measurement culture is not explained. However, analytical and number driven cultures are mentioned to involve professionals accustomed to evidence-based facts. In this way challenges may relate to DT principles and mindsets clashing with organizational culture and being a misfit with existing organizational processes and structures (Carlgren, 2016).

There is limited evidence on how outcomes of DT are measured, as DT is hard to measure as a single concept (Schmiedgen et al., 2015; Liedtka, 2017). Mentioned measurements that could relate to speed are: "Immediate results" (e.g. number of implemented projects based on DT sessions), and "Working culture" (e.g. Impact of DT within the organization measured by motivation, effectiveness, engagement and team collaboration) (Schmiedgen et al., 2015). However, traceable success stories in contrast to quantitative measurements may provide context and case specific-measurements showing a more realistic impact of the role of DT (Schmiedgen et al., 2015).

As DT seems to work as a standalone process to come up with concepts, it is used more as a support in later stages. In an organizational setting, DT is missing important functions to work as a "stand-alone end-to-end" innovation process. Mixing methods could therefore provide more value to new concepts. Tschimmel (2012) adds to this argument, stating that as innovation processes usually follow some kind of road map (Stage-Gate model etc.) managers could gradually introduce DT tools into existing stages of their innovation process. When studying DT in the Acceleration (exploitation) phase of innovation Carlgren (2016) states that engaging potential users through storytelling can be used to deliver a message, create recognition and show a need for change. In contrast to pushing technical information on users, DT involves storytelling, role-play, prototyping and human centric approaches that create an arena for users to experience needs and potential benefits. As previous literature emphasizes on visual and material practices dominating in the front end of innovation, the results from Carlgren (2016) study shows that DT practices plays a significant role also in the back-end.

\section{DIScussion}

To understand how DT may impact innovation speed, it has been relevant to gain insight into DTs main traits and usage. For the discussion part, it has been important to gain further insight and clarity on how DT may impact innovation speed specifically. Hence, common DT characteristics from the analysis have been categorized into three groups: visual, practical and contextual factors of DT.

\subsection{Visual factors}

DT is mentioned as a valuable method for visualization of ideas. Brainstorming through brainwriting and brainsketching is mentioned as variants of brainstorming to facilitate this. Since the authors' choice of DT techniques varies in terms of choice and purpose, it shows a variety of ways in which DT can be used.

As actors thought processes and routines might vary, DT techniques may reduce time spent to explain various concepts for other actors in a team setting, as well as reduce disagreements, which can delay the innovation process. Hence, increasing speed and gaining process flow might be acquired due to a deeper understanding and clarity between members within the process. Moreover, a more visual in contrast to verbal fashion of brainstorming representation of ideas, may reduce barriers related to not feeling comfortable with presenting thoughts. In this way, including more members to participate in the process may enhance innovation efficiency.

Reducing misunderstanding and creating meaning for other team members as to why something should be done is argued to facilitate empathy. Whether a product development setting involves end users or not, cultivating empathy from understanding may make it easier to reach agreements and continue with an idea. In this way, it may reduce unnecessary time spent on product development related discussions or disagreements. Being heard and understood may also result in higher levels of motivation within the project. Hence, impacting efforts and the amount of time and work spent towards the innovation 
positively. However, involving all members and their interests is essential, as having some team members dominating the process may result in some lacking behind or not feeling included.

In a situation involving the end user, a cultivation of empathy through understanding might also generate knowledge that make it easier for a product development team to get the right picture of what is needed and why. Hence, desired products may be created by avoiding unnecessary time and irrelevant resources and activity spent in the process. As DT involves co-creation, it may in this way be collectively reinforcing innovation capability, impacting speed within an organizational team setting.

As little research exists on strategic contributions of DT on innovative project management (Liedtka, 2014), DT might not only create value for the end user but for the organization as a whole. This is because as DT is thought to increase imaginative abilities and make sense of data that would otherwise be missed, it has an ability to show and capture details about individuals or their unique ideas. DT in this way may be used as a strategy to save time by revealing how valuable (or not) a partner is to contribute to a project. By knowing what needs, resources or activity a potential partner might have for the product development, DT might assess future conflict potential, inconsistencies and improvement areas as well as limit unnecessary time or resources spent in finding appropriate candidates. In this way, it may reduce uncertainty and risk associated with prioritizing various actors for the development process. Since brainstorming was argued by Seidel and Fixson (2013) to cause debates and in some cases conflict, and as more brainstorming could impact team effectiveness negatively, it may be a barrier to the speed of innovation. However, this issue is unclear due to the study being performed on student teams. It might thus be necessary to experiment with context (e.g. within industry and type of conflict) to find the best-suited amount of brainstorming and/or prototyping iterations to increase speed of product innovation capability.

\subsection{Practical factors}

Brainstorming focus on visualization of ideas for innovation and involves several visual idea generation techniques. Prototyping however, is the execution of these ideas into whole artifacts or personas which make it easier to see an idea or concept.

Testing solutions through rapid prototyping and failing early facilitates actor experience and knowledge. Being able to frame challenges and seeing what is to come therefore limits the chances of using time on creating inconclusive products, instead improving product accuracy from realistic input. However, reflecting a realistic product might in some cases require more expensive quality materials. The question of whether or not it pays off to prototype rather than create a final product thus becomes relevant. Furthermore, prototyping is said to drive out fear for failing as well as for the unknown. As fear could be one way to hinder innovation speed, DT could be a proactive way of building confidence through chaos; seeing novel ideas for the future through artifacts/personas. Hence, pre-experiencing solutions might save time, as obstacles can be hindered from taking shape.

Since prototyping emphasize on involvement and co-creation, it is argued to make way for understanding and quick communication in real time, which may speed up the process. A critical aspect is thus to avoid drifting of projects due to not being able to participate in the same space at the same time. Hence, being involved throughout the development process (e.g. digital journaling) may enhance involvement and knowledge flow, thus impacting innovation time positively.

Being part of someone's experience is argued to give rise to new ideas and perspectives, reducing reliance on past ideas or a present state of mind. Hence, hindering innovation creativity. By practicing DT, new information and combination of ideas may contribute to inspiration and imagination. In this way, DT tools may speed up the time it takes (e.g. process) to create any radical innovation, as well as impact the amount of innovations generated within a specific timeline. Hence, outcomes of DT on innovation speed may (similarly to "immediate results" and "working culture") be related to measuring the number of innovations and time used for its creation in various contexts. Additionally, behavioral characteristics (e.g. fear, motivation, empathy, engagement, team collaboration) should be measured together with the use of various DT tools. This may thus give an increased understanding of which DT tools and contexts leads to positive or negative outcomes related to speed. However, as events and behavior may change in time, tracing case-specific measurements may reduce risk and provide confidence in the positive impact of DT for speed.

DT is mentioned as a structured process to explore ill-defined problems (Mahmound-Jouini et al., 2016). In this way, DT tools may contribute to optimization of existing products or solving challenges within a team setting through understanding. However, by being able to view a situation or challenge from various points of view, facilitating clarity, meaning and empathy, it not only provides valuable 
input for product innovation but also facilitates a positive team dynamics, motivation and collaboration to be able to do so. Consequently, DT might be used as a method to create understanding and better cooperation as well as provide flexible thinking and imaginative abilities that help in thinking and acting more proactively. Hence, facilitating innovation within various stages of innovation as well as enhance company strategies for optimal innovative capability. In this way, impacting innovation speed through efficient innovation process flows.

As DT tool combinations may vary from project to project depending on the context, future research could focus on DT tools and their characterization from importance of a particular project. Hence, certain DT tools might not be necessary, and could save time in some cases. DT is thus understood as a process consisting of a series of "steps" with DT tools (which may vary) that facilitates action in order to speed up the innovation. Moreover, as being mindful of various hindrances to speed on team level is essential to speed up the innovation process, there is a need for empirical evidence on particular barriers to innovation to increase understanding of optimal utilization of DT in various situations.

\subsection{Contextual factors}

Factors that impact the value of DT can be seen as contextual factors. For this purpose, increasing the value of DTs usefulness to innovation speed is essential. Mixing methods or theory is thus mentioned as ways to do this. Looking at Carlgren (2016) study, as DT alone was not sufficient in later stages of innovation projects, it should be complemented with methods that suit the particular organization better. However, whether a mix will impact innovation speed, is not clear. What method combination generates the most efficient outcomes in terms of innovation speed, between which actors, based on situation and innovation phase thus remains to be investigated.

Impacting innovation speed within an organizational setting involves convincing its users of its value to be able to make way for a more rapid innovation pace. As DT workshops can visualize how products or methods may work or develop in various environments, it might provide employees with confidence of its value, thus improving perceptions. Traditional organizational cultures and perceptions might in this way be changed due to DTs ability to anticipate positive or negative situations. Hence, impacting employee's perception of DT in organizations, impacting weak design cultures and the need for process control. However, as resources to facilitate the competence of DT might be an issue, pre-experiencing its value to innovation speed could be advantageous.

Reflection and debate in connection with formal methods of DT (e.g. brainstorming) were argued to impact team performance. However, the results varied between innovation phases. Hence, there is a need to experiment with finding the right amount and timing for reflection and debate in connection to the various innovation phases and DT tools used in the process. Moreover, there is a need to look into forms of communication and various types of conflicts within a DT context and its connection to innovation performance. As such, reflection, debate and types of conflict as a result of practicing DT may impact speed negatively. Having experienced professionals to guide the process may thus be the best way to facilitate positive group dynamics and innovation efficiency.

As DT stress solution focused behavior, it is dependent on positive group states. Constructing a DT environment, which aims to facilitate understanding and openness to experimentation and individual ideas, may in this way inhibit chances of the process spiraling out of control. Moreover, if process conflicts (understood as disagreements), are understood to be acceptable within a DT context, it may create a positive cooperative environment. Consequently, facilitating enhanced communication and innovation within cross-disciplinary contexts.

\section{CONCLUSION}

This paper has given valuable insights as to how Design Thinking as a method may impact the speed (positively or negatively) of product innovation for complex technology-intense industries. The paper gives answers on the purpose, importance and challenges of visual, practical and contextual factors of DT that may apply to strategy formulation and increase (or decrease) innovation process flow. Hence, a framework is made from the main DT insights derived from the discussion. As innovation involves uncertainty and risk, fear among other barriers will have hindering effects on innovation performance. Capturing these barriers as well as being aware of DT tools and context is therefore essential in providing knowledge that build trust and confidence in new ideas. As DT emphasis on re-framing 
problems and gaining feedback from users or employees in a visual as well as practical way, degree of innovation capability can be decided from the creation of knowledge, understanding, meaning and empathy. However, an important prerequisite for success is taking into account the specific organizational context and the way DT is combined and implemented. In this way DT may be a sustainable way for organizations of the future to facilitate process and development opportunities beyond the initial creative phases of product development.

\section{REFERENCES}

Brown, T. and Martin, R. (2015), “Design for Action”, Harvard Business Review. Vol. 93 No. 9, pp. 56-64. Available at: https://hbr.org/2015/09/design-for-action (Accessed 11 Oct. 2018)

Brown, T. (2008), “Design Thinking”, [ebook] Harvard business review. Vol. 86 No. 6, pp. 84-141. Available at: https://churchill.imgix.net/files/pdfs/IDEO_HBR_DT_08.pdf (Accessed 8 Aug. 2018).

Buchanan, R. (1992), Wicked Problems in Design Thinking. [ebook] The MIT Press.Vol. 8 No. 2, pp. 5-21. https://doi.org/10.2307/1511637

Carlgren, L. (2016), "Design Thinking in innovation, in practice: The case of Kaiser Permanente", Euram Conference Proceedings. European Academy of Management, June 1-4, Paris, EURAM conference proceedings. European Academy of Management. Available at: http://publications.lib.chalmers.se/records/fulltext/247487/local_247487.pdf (Accessed 11 Oct. 2018)

Cooper, H. (1986), The Integrative Research Review, A Systematic Approach Sage Publications, Beverly Hills, 1984, p. 143, Educational Researcher, Vol. 15 No. 8, pp. 17-18. https://doi.org/10.3102/0013189X015008017

Cooper, H. M. (1988), Organizing knowledge synthesis: A taxonomy of literature reviews, Knowledge in Society, Vol. 1, 104-126. https://doi.org/10.1007/bf03177550

Cross, N. (2011), Understanding How Designers Think and Work. Berg Publishers, Oxford, U.K.

Jehn, K. A., Greer, L., Levine, S. and Szulanski, G. (2008), "The effects of conflict types, dimensions, and emergent states on group outcomes", Group Decision and Negotiation, Vol.17 No. 6, pp. 465-495. https://doi.org/10.1007/s10726-008-9107-0

Liedtka, J. (2014), "Perspective: Linking Design Thinking with Innovation Outcomes through Cognitive Bias Reduction", Journal of Product Innovation Management, Vol. 32 No. 6, pp. 925-938. https://doi.org/10.1111/jpim.12163

Lockwood, T. (2010), "Design Thinking”, Integrating Innovation, Customer Experience and Brand Value, Allworth Press, New York, NY.

Mahmound-Jouini, S. B., Midler, C., Silberzahn, P. (2016), "Contributions of Design Thinking in an innovation context”, Project management journal Vol. 47 No. 2, pp. 144-156. https://doi.org/10.1002/pmj.21577

O’Connor, G.C. and Ayers A.D. (2005), "Building a radical innovation competency", Research Technology Management, Vol. 48 No. 1, 23-31. https://doi.org/10.1080/08956308.2005.11657292

Meinel, C., Leifer, L., Plattner, H. (2011), Design Thinking- Understand-Improve-Apply, Springer, Berlin. https://doi.org/10.1007/978-3-642-13757-0

Seidel, V. P. and Fixson S. K., (2013), “Adopting "Design Thinking” in novice multidisciplinary teams: The application and limits of design methods and reflexive practices", Product innovation management, Vol. 30 No. 6. https://doi.org/10.1111/jpim.12061

Schmiedgen, J., Spille, L., Köppen, E., Rhinow, H., Meinel, C. (2016), "Measuring the Impact of Design Thinking", Design Thinking Research: Making Design Thinking Foundational. Plattner, H., Meinel C. and Leifer, L. Springer International Publishing, Cham, pp. 157-170 https://doi.org/10.1007/978-3-319-19641-1_11

Simon, H. (1969), The sciences of the artificial (Vol. 136, MIT science). M.I.T, Cambridge, Mass.

Simon, H. A. (1996), The sciences of the artificial, MIT Press, Cambridge, Mass.

Tschimmel, K. (2012), “Design Thinking as an effective Toolkit for Innovation”, In: Proceedings of the XXIII ISPIM Conference: Action for Innovation: Innovating from Experience. Barcelona. ISBN 978- 952-256243-0 Performance. IEEE Transactions on Engineering Management, Vol. 59 No. 1, pp. 4-19.

Wallace, M. and Wray, A. (2011), Critical reading and writing for postgraduates. 2nd ed., Sage, London.

Weinreich, N. (2011), Hands-on Social Marketing: A Step-by-Step Guide to Designing Change for Good, 2nd ed., SAGE Publications, Inc, Thousand Oaks. http://doi.org/10.4135/9781452224879 